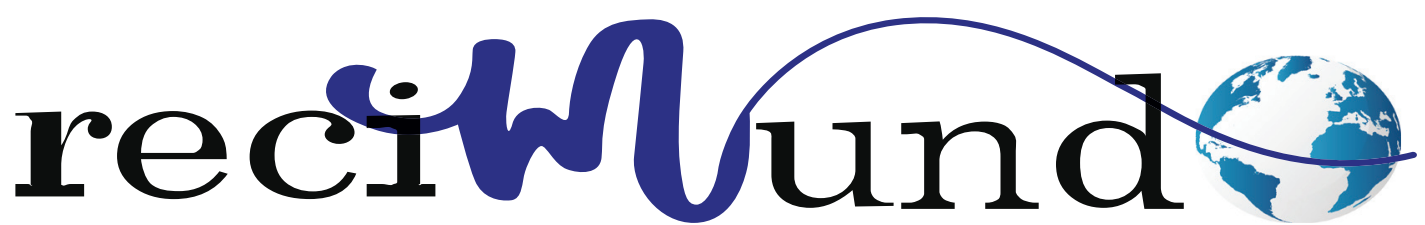

Revista Científica Mundo de la Investigación y el Conocimiento

DOI: 10.26820/recimundo/4.(1).enero.2020.423-433

URL: http://recimundo.com/index.php/es/article/view/771

EDITORIAL: Saberes del Conocimiento

REVISTA: RECIMUNDO

ISSN: 2588-073X

TIPO DE INVESTIGACIÓN: Artículo de Revisión

CóDIGo UNESCO: 3201 Ciencias Clínicas; 3202 Epidemiología

PAGINAS: $423-433$

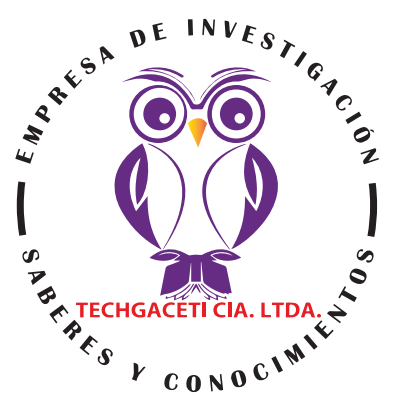

\title{
Manejo de hiperlipidemia familiar combinada
}

\section{Management of combined familial hyperlipidemia}

\section{Tratamento da hiperlipidemia familiar combinada}

Veronica Vanessa Vicuña Terán'; Pedro Alejandro Chumbi Zumba2;

Jefferson Leonardo León García3; Karen Lissette Sánchez Pincay4

RECIBIDO: 18/09/2019 ACEPTADO: 29/10/2019 PUBLICADO: 31/01/2020

1. Médica; Investigador Independiente; Guayaquil, Ecuador; romi422@live.com; (D) https://orcid.org/0000-00021191-5607

2. Médica General; Investigador Independiente; Guayaquil, Ecuador; peterchumbi@hotmail.com; (DD https://orcid.org/0000-0002-0811-2029

3. Médico; Investigador Independiente; Guayaquil, Ecuador; jeffo.leo.grc@gmail.com; (D) https://orcid.org/00000003-3383-0783

4. Médico; Investigador Independiente; Guayaquil, Ecuador; karensanpi92@gmail.com; (D) https://orcid.org/00000003-0434-2123

\author{
CORRESPONDENCIA \\ Veronica Vanessa Vicuña Terán \\ romi422@live.com \\ Guayaquil, Ecuador
}

() RECIMUNDO; Editorial Saberes del Conocimiento, 2020 


\section{RESUMEN}

La hiperlipidemia familiar combinada (HFC), es la más frecuente de las dislipidemias familiares de origen genético, siendo este, un factor de riesgo para el aumento de la enfermedad arterial coronaria prematura. La hiperlipidemia familiar combinada se origina por la expresión variable de hipercolesterolemia y/o hipertrigliceridemia, según, la aplicación de estudios genéticos en varias poblaciones ha reconocido regiones cromosómicas que determinan la concentración de la apolipoproteína B y la diversidad en la actividad o concentración de varios factores nucleares (USF1, TCF7L2, HNF4alfa), que son importante en la fisiopatología de la HLFC y variantes genéticas idóneas para el desarrollo de este padecimiento. Esta investigación analiza las bases genéticas de la dislipidemia común y resaltar el avance en la identificación de genes susceptibles para la hiperlipidemia combinada en familias. Se necesitan, al menos tres familiares directos para confirmar el diagnóstico. Considerándose que, es una enfermedad oligogénica es posible la identificación de los genes involucrados con los recursos ahora existentes. El diagnóstico se hace mediante procedimientos clínicos, con referencia a la variabilidad del fenotipo lipídico y la historia familiar de hiperlipidemia. Usualmente, se relaciona con diabetes mellitus tipo 2, hipertensión arterial y obesidad central. Los pacientes diagnosticados con hiperlipidemia familiar combinada (HFC), tienden a riesgo cardiovascular alto y el objetivo terapéutico es un colesterol-LDL $<100 \mathrm{mg} / \mathrm{dl}$, y $<70 \mathrm{mg} / \mathrm{dl}$ en $\mathrm{presencia}$ de enfermedad cardiovascular establecida o diabetes mellitus.

Palabras clave: Hiperlipidemia; Diabetes Mellitus Tipo 2; Hipertensión; Tratamiento Hipolipemiante; Poligénica; Dislipidemia; Hipercolesterolemia; Hipertrigliceridemia.

\section{ABSTRACT}

Combined familial hyperlipidemia (HFC) is the most frequent of family dyslipidemias of genetic origin, this being a risk factor for the increase in premature coronary artery disease. Combined familial hyperlipidemia is caused by the variable expression of hypercholesterolemia and / or hypertriglyceridemia, according to the application of genetic studies in several populations has recognized chromosomal regions that determine the concentration of apolipoprotein B and the diversity in activity or concentration of several factors Nuclear (USF1, TCF7L2, HNF4alfa), which are important in the pathophysiology of HLFC and genetic variants suitable for the development of this condition. This research analyzes the genetic basis of common dyslipidemia and highlights the progress in the identification of susceptible genes for combined hyperlipidemia in families. At least three direct relatives are needed to confirm the diagnosis. Considering that, it is an oligogenic disease, it is possible to identify the genes involved with the now existing resources. The diagnosis is made by clinical procedures, with reference to the variability of the lipid phenotype and the family history of hyperlipidemia. Usually, it is related to type 2 diabetes mellitus, high blood pressure and central obesity. Patients diagnosed with combined familial hyperlipidemia (HFC) tend to have a high cardiovascular risk and the therapeutic objective is an LDL-cholesterol <100 $\mathrm{mg} / \mathrm{dl}$, and <70 $\mathrm{mg} / \mathrm{dl}$ in the presence of established cardiovascular disease or diabetes mellitus.

Keywords: Hyperlipidemia; Diabetes mellitus type 2; Hypertension; Hypolipidemic treatment; Polygenic; Dyslipidemia; Hypercholesterolemia; Hypertriglyceridemia

\section{RESUMO}

A hiperlipidemia familiar combinada (HFC) é a dislipidemias familiares de origem genética mais frequente, sendo este um fator de risco para o aumento da doença arterial coronariana prematura. A hiperlipidemia familiar combinada é causada pela expressão variável de hipercolesterolemia e / ou hipertrigliceridemia, de acordo com a aplicação de estudos genéticos em várias populações, reconheceu regiões cromossômicas que determinam a concentração de apolipoproteína B e a diversidade na atividade ou concentração de vários fatores Nucleares (USF1, TCF7L2, HNF4alfa), importantes na fisiopatologia do HLFC e variantes genéticas adequadas para o desenvolvimento dessa condição. Esta pesquisa analisa a base genética da dislipidemia comum e destaca o progresso na identificação de genes suscetíveis à hiperlipidemia combinada em famílias. São necessários pelo menos três parentes diretos para confirmar o diagnóstico. Considerando que, é uma doença oligogênica, é possível identificar os genes envolvidos com os recursos atualmente existentes. O diagnóstico é feito por procedimentos clínicos, com referência à variabilidade do fenótipo lipídico e ao histórico familiar de hiperlipidemia. Geralmente, está relacionado ao diabetes mellitus tipo 2, pressão alta e obesidade central. Pacientes diagnosticados com hiperlipidemia familiar combinada (HFC) tendem a apresentar alto risco cardiovascular e o objetivo terapêutico é um colesterol LDL $<100 \mathrm{mg} / \mathrm{dl}$ e $<70 \mathrm{mg} / \mathrm{dl}$ na presença de doença cardiovascular estabelecida ou diabetes mellitus.

Palavras-chave: Hiperlipidemia; Diabetes Mellitus Tipo 2; Hipertensão; Tratamento hipolipidêmico; Poligênico; Dislipidemia; Hipercolesterolemia; Hipertrigliceridemia. 


\section{Introudcción}

La hiperlipidemia familiar combinada (HFC) es el trastorno heredado más común del metabolismo lipídico relacionado con hiperlipidemia mixta y enfermedad cardiovascular (ECV) prematura. Fue definida por Goldstein en 1973 al realizar estudios a familias de supervivientes de infarto de miocardio (IM), (Goldstein, Schrott, Hazzard, \& Bierman, 1973). Ocasionalmente, esta patología puede manifestarse en niños, si bien es cierto, la hipercolesterolemia y/o hipertrigliceridemia se manifiesta a partir de la segunda década de vida de los pacientes, con una modificación fenotípica intraindividual e interindividual, teniendo como resultado, la interacción entre factores genéticos y ambientales como la alimentación, el aumento del peso corporal, el consumo de alcohol y el tiempo de evolución (Porkka, y otros, 1997) (Gaddi, Cicero, Poli, \& Paoletti, 2007).

Se indica que, la hiperlipidemia familiar combinada (HFC) se relaciona con varias anormalidades metabólicas, como hipertensión arterial (HTA), resistencia a la insulina, diabetes mellitus (DM) tipo 2, obesidad central, esteatosis hepática y síndrome metabólico (Brouwers, y otros, 2007) (Skoumas, y otros, 2007). De hecho, visualizaron que varios miembros de las familias tenían aumentos moderados de colesterol y/o triglicéridos y que el patrón de herencia era similar a autosómico dominante. (Keulen, Kruijshoop, Schaper, \& et al, 2002). Debe señalarse que, esta patología es la segunda causa de muerte en países desarrollados por sus factores de riesgos cardiovasculares.

Por otra parte, la enfermedad tiene la particularidad; de concentraciones altas de la apoproteína B, el aumento de colesterol-LDL y/o triglicéridos es regular (pocas veces arriba de $300 \mathrm{mg} / \mathrm{dL}$ ), el predominio de las subclases pequeñas y densas entre las lipoproteínas de baja densidad (LDL), la falta de xantomas, y la historial familiar de cardiopatía isquémica. Observándose continuamente, fluctuaciones espontáneas en las concentraciones de colesterol y triglicéridos. Es por eso que, se puede encontrar alternancia entre hipercolesterolemia, hipertrigliceridemia, una dislipidemia mixta o incluso la regularización de la concentración de los lípidos séricos en el mismo individuo sin que exista ningún cambio en sus condiciones clínicas.

En el año 1987, se unificaron criterios para su diagnóstico, más sin embargo, estos criterios son motivo de controversia aún. (Goldstein J., Schrott, Hazzard, Bierman, \& Motulsky, 1973). Algunos autores requieren la presencia de más de un familiar con niveles anormales de triglicéridos o con concentraciones altas de colesterol, preferiblemente con ambos defectos. Por tal motivo, el diagnóstico con certidumbre se establecer cuando en el estudio de la familia se extiende a cuantos miembros sean posibles.

\section{Metodologia}

La revisión que se presenta en este artículo es de tipo documental, la recopilación de la información se ha realizado a través de una investigación con análisis bibliográfico y de estudios metanalisis encontrados a través de medios Electrónicos, en donde hay disponible material de consulta sobre manejo de hiperlipidemia familiar combinada. Aquí se consideran los aportes más importantes de ciertos autores, con el objetivo de dejar abierta la investigación para futuros casos que den soporte a nuevos estudios.

\section{Resultados}

La hiperlipidemia familiar combinada (HFC); es un trastorno hereditario que provoca niveles de colesterol y triglicéridos altos en la sangre, relacionado con hiperlipidemia mixta y enfermedad cardiovascular (ECV) prematura. El origen de la enfermedad se desconoce. Sin duda, hay un incremento de producción hepática de la apoproteína B (siendo el resultado de las lipoproteínas de muy baja densidad conocidas también

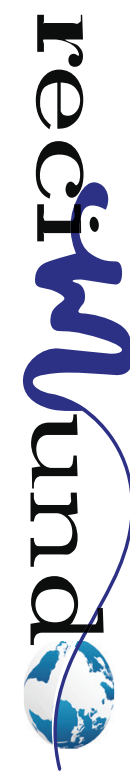


como VLDL's), (Grundy, Chait, \& Brunzell, 1987). Esto se debe, a un aumento de la concentración de ácidos grasos libres en plasma, incrementando la manifestación del gen de la apoproteína B y estimulando la secreción de las VLDL. (Erkelens, 1998).

La hiperlipidemia familiar combinado (HFC) es la patología más común de las dislipidemias de etimiologia genética, con una frecuencia de $0,5 \%$ a $2 \%$ en la población general y de $14 \%$ entre los pacientes con enfermedad cardiovascular prematura (Davignon \& Genest, 1998) (Aguilar-Salinas, y otros, 2001). La HFC participa en el 30-50\% de los casos con cardiopatía isquémica de genesis familiar. Debido a los criterios establecidos de diagnósticos actuales, no se conoce con certeza su predominio en estudios con representación poblacional. Sin embargo, sus variedades más frecuentes (la hipertrigliceridemia aislada y la hiperlipidemia mixta) se presentan en mayor porcentaje de adultos que viven en zonas pobladas (Arner, 1997). Al emplear la caracterización propuesta por Sniderman, la prevalencia fue 8,1\% en la Encuesta Nacional de Enfermedades Crónicas de México (1993-1994). Es la hiperlipidemia más comun en la mayoría de las clínicas de lípidos (Aguilar-Salinas, Huertas, Tusie, Gómez-Pérez, \& Rull, 2002) Su índice aterogénico ha sido comprobado por dos estudios recientes (Mc Neely, y otros, 2001) (Austin, McKnight, \& Edward, 2000).

Su diferenciacion fue realizada por Goldstein y Brown en 1973 después de sistematizar más de 50 familias con historial de cardiopatía isquémica prematura. Fue observada como la hiperlipidemia más frecuente en los pacientes con un infarto agudo al miocardio que han sobrevivido. Reportaron que múltiples miembros de las familias tenían colesterol y/o triglicéridos con elevaciones de moderadas a altas, y que el patrón de herencia era compatible con un modelo de dominancia genética (Goldstein J. , Schrott, Hazzard, Bierman, \& Motulsky, 1973). En 1987 se clasificaron los criterios para el diagnóstico, sin embargo, estos son motivo de controversia actualmente (Grundy, Chait, \& Brunzell, 1987). Definida por tres fenotipos lipídicos: hipercolesterolemia, hipertrigliceridemia ó padece las dos. Se requiere la presencia de más, de un familiar con concentraciones altas de colesterol, con niveles anormales de triglicéridos o para mayor valoración con ambos defectos. Por ello, para concluir el diagnóstico con certidumbre es requerido el estudio de la mayoría de miembros de la familia que sean posibles.

Otras características de la enfermedad permiten sospechar su presencia. Cuando las concentraciones de la apoproteína B se encuentran por encima del 90\%. Este valor corresponde a $108 \mathrm{mg} / \mathrm{dL}$ en hombres y 99 $\mathrm{mg} / \mathrm{dL}$ en mujeres (Valles, y otros, 2002). La elevación de colesterol- LDL y/o de los triglicéridos es moderada (por debajo de $300 \mathrm{mg} / \mathrm{dL}$ ), sin embargo al relacionarse con otras causas de dislipidemia pueden alcanzar niveles extremadamente altos de colesterol y/o triglicéridos (>300 mg/dL). Un porcentaje alto de los casos tienen predominio las lipoproteínas de baja densidad (LDL). Esta particularidad es una de las explicaciones de la mayor aterogenicidad de la HFC, ya que un factor de riesgo independiente para sufrir eventos cardiovasculares son las subclases pequeñas y densas de las LDL's.

Por ello, la alternancia entre hipercolesterolemia, hipertrigliceridemia, una dislipidemia mixta o incluso normalización de la concentración de los lípidos séricos se puede encontrar en el mismo individuo, sin que cambie en sus condiciones clínicas. Se desconocen las causas de estas fluctuaciones. Sin embargo, el riesgo cardiovascular no desaparece con la normalización espontánea del perfil de lípidos. McNeely y col. (Mc Neely, y otros, 2001) demostraron que los sujetos con lípidos normales que habían sido considerados como afectados, teniendo una tasa de eventos cardiovasculares a 20 años, parecidos a las personas diagnosticadas con hiperlipidémia. 
En la mayoría de los casos es posible concluir la resistencia a la insulina y su frecuencia es mayor en pacientes diabéticos. Por tal motivo, es común encontrar la hiperlipidemia primaria en pacientes con diabetes sobrevivientes a un infarto del miocardio, en un $64,7 \%$ de los casos coexiste con el síndrome metabólico. Un porcentaje similar fue informado en una de investigación mexicana (Zamora, Aguilar-Salinas, \& Gomez-Pérez, 2004). La cantidad de grasa intraabdominal, la sensibilidad a la insulina, las concentraciones de lípidos sanguíneos y de la apoproteína B no pueden ser explicadas en estudios transversales. De estas evidencias, al comparar el coeficiente de regresión de las variables antes mencionadas y la HFC se presentan diferencias significativas entre la población general.

Es difícil catalogar con certeza los casos anormales en niños de familias afectadas. Sus concentraciones en los perfiles lipídicos son mayores que la observada en niños de familias sin HFC; sin embargo, la diferencia no permite identificar los casos afectados.

\section{Causas}

La hiperlipidemia combinada familiar es el trastorno genético más común que aumenta las grasas en la sangre y puede causar ataques cardíacos miocardios precoces, hipertensión arterial (HTA), resistencia a la insulina, diabetes mellitus (DM) tipo 2, obesidad central, esteatosis hepática y síndrome metabólico La diabetes, el alcoholismo y el hipotiroidismo empeoran esta afección. Los factores de riesgo incluyen antecedentes familiares de colesterol alto y arteriopatía coronaria temprana.

\section{Síntomas}

Es posible que en los primeros años no se presenten síntomas. Cuando los síntomas aparecen, pueden incluir: dolor de pecho (angina) u otros signos de enfermedad coronaria; se pueden presentar en una edad joven, calambres en una o ambas pantorrillas al caminar, llagas en los dedos de los pies que no sanan. Síntomas repentinos similares a un accidente cerebrovascular, tales como problemas para hablar, caída de un lado de la cara, debilidad de un brazo o una pierna y pérdida de equilibrio.

Con respecto, a las personas con esta condición pueden desarrollar colesterol alto o niveles altos de triglicéridos en la adolescencia. La patologia puede ser diagnosticada cuando los pacientes tienen entre 20 y 30 años. Así mismo, sus niveles de hiperlipidemia siguen siendo altos a lo largo de toda la vida.

\section{Característica hiperlipidemia familiar combinada}

Hipercolesterolemia (fenotipo II), hipertrigliceridemia (fenotipo IV) o ambas (fenotipo II b). Se requiere la presencia de al menos un familiar con concentraciones altas de colesterol, otro con niveles anormales de triglicéridos y otro con ambos defectos. Otras características de la enfermedad permiten sospechar su presencia, las concentraciones de la apoproteína B.

\section{Diagnostico}

Los criterios diagnósticos de la HFC fueron descritos hace más de 20 años, a pesar de ello, siguen siendo motivo de controversia. La falta de un indicador genético ha producido diversos criterios de diagnósticos y múltiples variantes de los criterios originales. Los investigadores han basado el diagnóstico en los valores de colesterol, triglicéridos y de colesterol HDL; otros han valorado los niveles de la apolipoproteína B debido a la relación de esta proteína en la patogénesis de la HFC. Además, diferentes niveles de concentración se han empleados para considerar como valores anormales de los lípidos séricos. La definición más comunmente utilizada es un valor de colesterol o triglicéridos mayor del 90 percentil y al menos dos familiares con HFC. diversos autores recalcan la necesidad de incluir en los criterios diagnósticos la apolipoproteína B. 
Recientes estudios han incluido cohortes de pacientes con HFC, en los que utilizaron a la apolipoproteína B en el diagnóstico y tuvieron menor dispersión en los lípidos séricos comparado con los diagnóstico basados en la concentración de colesterol y de triglicéridos (Aguilar-Salinas, y otros, 2004). Un consenso organizado por investigadores europeos y canadienses, con la finalidad de homologar los criterios diagnósticos, modificó la definición. concluyeron que el diagnóstico se debe establecer en presencia de valores altos de apolipoproteína B ( 120 $\mathrm{mg} / \mathrm{dL}$ ) y una concentración de triglicéridos mayor de 130 mg/dL (Sniderman, y otros, 2002). Esta definición difiere del perfil clínico inicialmente propuesto para la HFC.

Las concentraciones altas de la apolipoproteína B (por arriba de la percentila 90) serán usadas como prueba confirmatoria. En los casos en que los datos colectados y los elementos requeridos no son suficiente para establecer el diagnóstico, la existencia de hipertrigliceridemia más la concentración alta de apolipoproteína B y/o variaciones espontáneas del perfil de lípidos, son datos que deben despertar sospechas en el diagnóstico. De estas evidencias, es necesario informar al médico de primer contacto sobre la importancia de solicitar, a todos los familiares de primer grado del paciente, la concentración de los lípidos sanguíneos. Es el mejor método para hacer el diagnóstico de la HFC. Los estudios a los familiares permitirá identificar nuevos casos, e iniciar el tratamiento antes de la aparición de los síntomas.

Finalmente, en los pacientes con HFC, con niveles de colesterol y triglicéridos alto en la adolescencia o en pacientes entre 20 y 30 años, tienen un mayor riesgo de sufrir arteriopatía coronaria precoz y ataques cardíacos. Además ambos niveles (colesterol y triglicéridos) se mantiene altos a lo largo de toda la vida. Por esta razón, la tasa de obesidad es mayor y son más propensas a sufrir intolerancia a la glucosa.
Entre los factores que permiten el diagnóstico:

- Herencia autosómica Dominante.

- Prevalencia 0,5-1\%.

- Puede detectarse en edad infantil, pero su expresión clínica es aprox. en la $3^{a}$ década.

- Fenotipo Ila, IIb, o IV en varios miembros de la familia que pueden variar en el tiempo en un mismo paciente.

- Producción excesiva de Apo B 100, pero mecanismo desconocido.

- Síntesis excesiva de VLDL.

- Con frecuencia lipolisis defectuosa.

- Pueden aparecer LDL de pequeño tamaño.

- Posible aumento de IDL y VLDL.

- Frecuente asociación con obesidad, HTA, hiperuricemia e insulín resistencia.

- Frecuente: Triglicéridos 200-400 mg/dl. 
Tabla 1. Criterios para el diagnósticos de la hiperlipidemia familiar combinada

\begin{tabular}{|c|c|}
\hline \multicolumn{2}{|l|}{ 1. Persona afectada } \\
\hline \multirow{3}{*}{ - En adultos: } & colesterol total > $240 \mathrm{mg} / \mathrm{dl}$ \\
\hline & colesterol LDL > 160 mg/dl \\
\hline & triglicéridos > 200 mg/dl \\
\hline \multirow{3}{*}{ - En menores de 20 años: } & colesterol total > $200 \mathrm{mg} / \mathrm{dl}$ \\
\hline & colesterol LDL > 130 mg/dl \\
\hline & triglicéridos > 120 mg/dl \\
\hline \multirow{4}{*}{$\begin{array}{l}\text { - Descartar causas secundarias: índice de masa } \\
\text { corporal }\end{array}$} & $>35 \mathrm{~kg} / \mathrm{m} 2$ \\
\hline & $\mathrm{HbA} 1 \mathrm{c}>10 \%$ \\
\hline & hipotiroidismo no controlado \\
\hline & etilismo (> $40 \mathrm{~g}$ alcohol/día) \\
\hline \multicolumn{2}{|l|}{ 2. Familia afectada } \\
\hline \multirow{5}{*}{$\begin{array}{l}\text { - Dos o más miembros de primer grado (padres, } \\
\text { hermanos, hijos) }\end{array}$} & afectados de hiperlipidemia mixta \\
\hline & combinaciones de fenotipos, \\
\hline & hipercolesterolemia pura (Ila) \\
\hline & hiperlipidemia mixta (Ilb) \\
\hline & hipertrigliceridemia (IV) \\
\hline \multirow{4}{*}{ - Se excluyen familias } & con xantomas tendinosos \\
\hline & y/o cifras de c-LDL > 300 mg/dl \\
\hline & en 2 o más familiares de primer grado \\
\hline & con fenotipo lia \\
\hline \multirow{3}{*}{ - Historia familiar de enfermedad cardiovascular } & ateroesclerótica prematura (<60 años) \\
\hline & 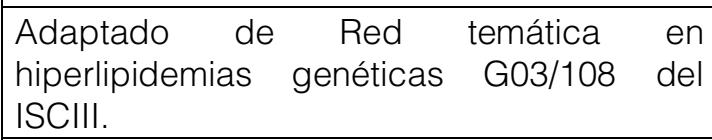 \\
\hline & $\begin{array}{l}\text { La historia familiar de ECV prematura no es } \\
\text { un criterio estricto de HFC }\end{array}$ \\
\hline
\end{tabular}

Fuente: Autores 2020

\section{Pruebas y exámenes}

Se realizan exámenes de sangre para verificar los niveles de colesterol y triglicéridos. Los exámenes mostrarán:

- Aumento del colesterol LDL.

- Disminución del colesterol HDL.

- Aumento de los triglicéridos.

- Aumento de la apolipoproteína B100.

En la actualidad, están disponibles las pruebas genéticas para un tipo de hiperlipidemia combinada familiar.

\section{Manejo de la hiperlipidemia familiar com- binada}

El primer paso es realizar cambios en el estilo de vida, es decir, la alimentación del individuo. Muchas veces, las personas intentan cambios en la dieta por algunos meses antes que el médico le indique medicamentos. La modificación en la dieta incluye la disminución en la cantidad de grasa saturada y azúcar refinado.

Cambios Recomendados:

- Ingerir menos carne de res, pollo, cerdo y cordero. 
- Sustituir los productos lácteos ricos en grasa por los bajos en grasa.

- Evitar los bizcochos empacados y los productos horneados que contengan grasas trans.

- Reducir el colesterol, eliminando las yemas de huevo y las vísceras.

Finalmente, se aconseja la asesoría nutricional para ayudar a los pacientes a hacer esta adaptación en sus nuevos hábitos alimenticios. Ejercitarse constantemente, logrando pérdida de peso ayudando así, a bajar los niveles de colesterol.

\section{Medicamentos}

Evidentemente, cuando las modificaciones en el estilo de vida no corrigen los niveles de colesterol, el paciente tiene la posibilidad de padecer cardiopatía ateroesclerótica, el médico le indica que tome medicamentos. Es por eso que, existen diferentes tipos de fármacos para contribuir a bajar los niveles de colesterol.

Los fármacos ayudan a obtener los niveles de lípidos óptimos. Sin embargo, hay algunos que se utilizan para bajar el colesterol LDL, otros, fármacos son útiles para bajar los triglicéridos, por otra parte, otros fármacos ayudan a aumentar el colesterol HDL.

Los fármacos más efectivos y utilizados con mayor resultado para tratar el colesterol LDL alto se denominan estatinas. Éstas incluyen lovastatina (Mevacor), pravastatina (Pravachol), simvastatina (Zocor), fluvastatina (Lescol), atorvastatina (Lipitor), rosuvastatina (Crestor) y pitivastatina (Livalo).

\section{No obstante, existen otros medicamentos hipocolesterolemiantes}

- Resinas secuestradoras de ácidos biliares.

- Ezetimiba.

- Fibratos (como gemfibrozilo y fenofibrato).
- Ácido nicotínico.

- Inhibidores de PCSK9, como alirocumab (Praluent) y evolocumab (Repatha).

Estos fármacos representan la nueva generación para tratar el colesterol alto.

\section{Tratamiento de la hipertensión arterial en la hiperlipidemia familiar combinada}

La hipertensión arterial (HTA) usualmente se relaciona a la hiperlipidemia familiar combinada (HFC). Con respecto, a los pacientes con hiperlipidemia familiar combinada (HFC) son pacientes de alto o muy alto RCV, considerando, el tratamiento farmacológico al momento del diagnóstico en todos los pacientes con HTA grado 102 , independientemente el cambio de estilo de vida. El tratamiento farmacológico se debe suministrar de forma inmediata en los pacientes con HTA.

Es recomendable, comenzar el tratamiento con dosis bajas del fármaco o fármacos, proyectando una disminución gradual de la presión arterial (PA). Se tiene que utilizar fármacos de acción retardada que permitan solo una dosis diaria. El control de la PA con monoterapia solo se consigue en el $30 \%$, en consecuencia, habrá que aplicar terapia combinada. Es recomendable el empleo de fármacos asociados para mejorar el cumplimiento terapéutico (Mancia, y otros, 2013). 


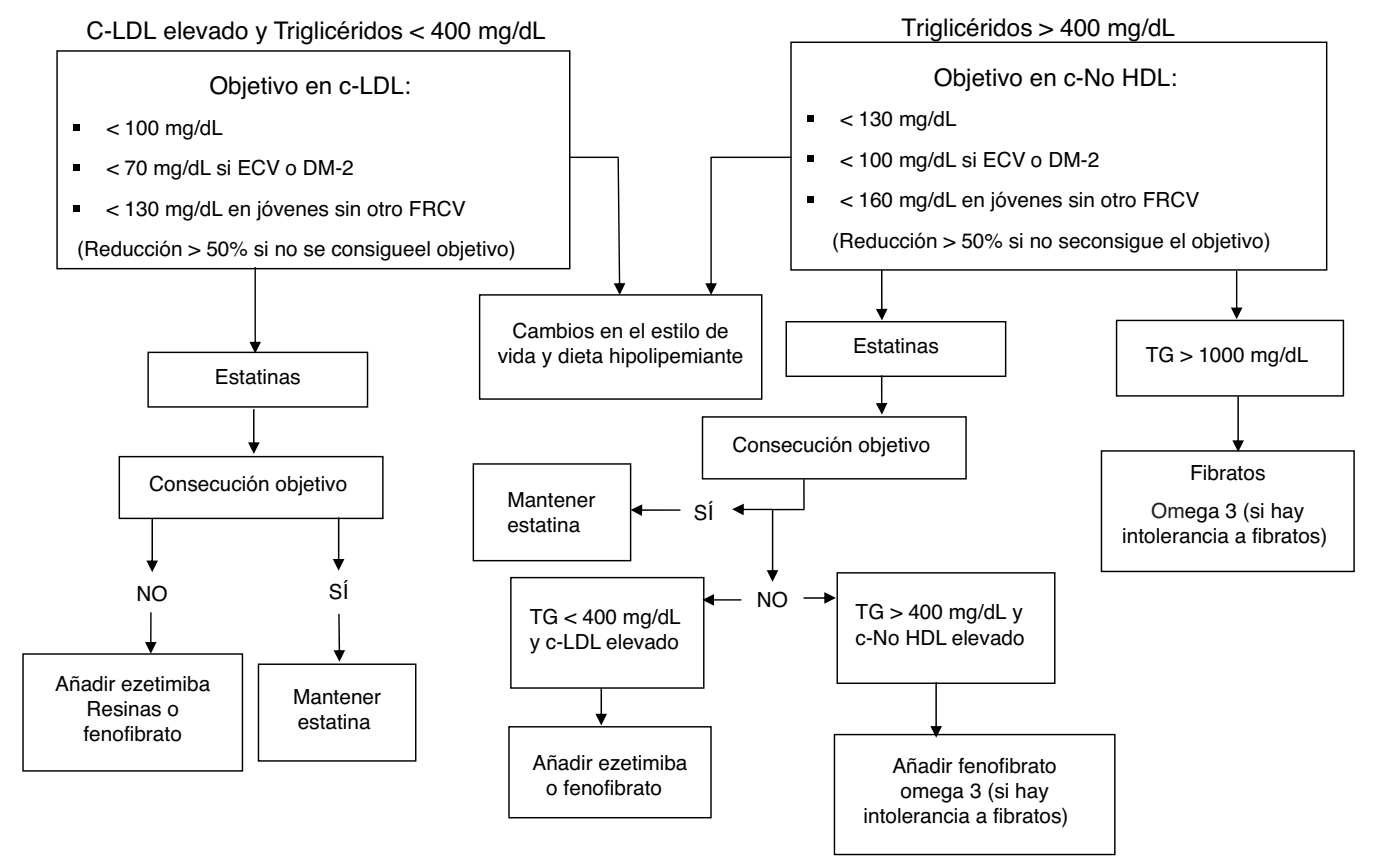

Gráfica 1. Algoritmo terapéutico en adultos con hiperlipidemia familiar combinada (HFC)

Fuente: (Mata, Alonso, Ruíz-Garcia, Díaz-Díaz, \& González, 2020)

\section{Pronóstico}

La evolución del paciente puede variar dependiendo de:

- El diagnóstico temprano de la patología.

- El inicio temprano del tratamiento recomendado por el médico.

- Realizar el tratamiento siguiendo instrucciones médicas.

En consecuencia, el paciente esta propenso a sufrir un ataque cardíaco o un accidente cerebrovascular sino se administra algún tratamiento, causando la muerte prematura.

De igual manera, con medicamentos, hay pacientes que continúan teniendo niveles de lípidos altos que aumentan el riesgo de ataque cardíaco.

\section{Posibles complicaciones}

Las complicaciones pueden incluir:

- Cardiopatía ateroesclerótica temprana.

- Ataque cardíaco.
- Accidente cerebrovascular.

Cuando se presenta dolor en el pecho $u$ otras características de advertencia de un ataque cardíaco acudir a un centro de asistencia médica primaria.

\section{Prevención}

Mantener un régimen alimenticio bajo en colesterol y grasa saturada para lograr controlar los niveles de LDL en personas con alto riesgo. Si algún miembro en su familia padece esta enfermedad, es recomendable realizar pruebas genéticas a usted y a sus hijos. De igual manera, los niños pequeños pueden tener una hiperlipidemia leve. Es importante controlar otros factores de riesgo de ataques cardíacos tempranos, como el tabaquismo.

\section{Conclusion}

La HFC es la hiperlipidemia primaria más frecuente de estas patologías. El riesgo cardiovascular presente ha sido demostrado en estudios prospectivos. A pesar de ello, la información que tiene el médico de primer contacto y el público sobre esta condición

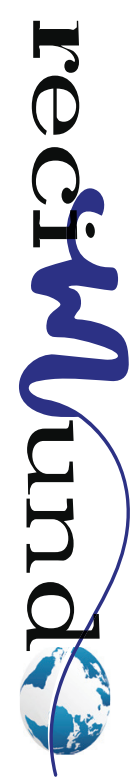


de salud es insuficiente. La HFC es un modelo de dislipidemia en el que la investigación clínica y bioquímica puede lograr mejores beneficios, en este orden de ideas, es necesario instar al médico de primer contacto sobre lo imprescindible de solicitar, a todos los familiares en primer grado del paciente, la concentración de los lípidos sanguíneos, este constituye el mejor método para hacer el diagnóstico de la HFC, además, los estudios a los familiares permitirá identificar nuevos casos, e iniciar el tratamiento antes de la aparición de los síntomas.

Por los estudios recientes y el avance ocurrido en las dos décadas más recientes, se han logrado identificar regiones cromosómicas que determinan la concentración de la apolipoproteína B y se concluyó que diferencias en la actividad o concentración de diversos factores nucleares determinan la fisiopatología de la HFC.

Finalmente, esta revisión propone la necesidad de realizar nuevos estudios genéticos en asociación con múltiples marcadores bioquímicos y en biopsias de grasa o hígado, para sistematizar una caracterización completa de la fisiopatología, para que la HFC pueda ser tratada en forma oportuna.

\section{Bibliografía}

Aguilar-Salinas, C., Huertas, A., Tusie, M., Gómez-Pérez, F., \& Rull, J. (2002). Hiperlipidemia familiar combinada. Endocrinol Nutr, 10, 58-62.

Aguilar-Salinas, C., Olaiz, G., Valles, V., Ríos, J., Gómez Pérez, F., Rull, J., . . Sepúlveda, J. (2001). High prevalence of low HDL cholesterol concentrations and mixed hyperlipidemia in a Mexican nation wide survey (Vol. 42). J Lipid Res.

Aguilar-Salinas, C., Zamora, M., Gómez-Díaz, R., Mehta, R., Gómez-Pérez, F., \& Rull, J. (2004). Familial combined hyperlipidemia: controversial aspects of its diagnosis and pathogenesis (Vol. 4). Semin Vasc Med.

Arner, P. (1997). Is familial combined hyperlipidaemia a genetic disorder of adipose tissue? (Vol. 8). Curr Opin Lipidol.

Austin, M., McKnight, B., \& Edward, K. (2000). Cardiovascular disease mortality in familial forms of hypertriglyceridemia: A 20 year prospective study (Vol. 101). Circulation.

Brouwers, M., Van Greevenbroek, M., Vermeulen, V., Van Lin, J., Van der Kallen, C., \& De Bruin, T. (2007). Five-year follow up of waist circumference, insulin and ALT levels in familial combined hyperlypidaemia (Vol. 113). Londres: Clin Sci.

Davignon, J., \& Genest, J. (1998). Genetics of lipoprotein disorders (Vol. 27). Endoc Metab Clin North Am.

Erkelens, D. (1998). Metabolic basis for hypertriglyceridaemia in familial combined hyperlipidaemia (Vol. 19). Eur Heart J.

Gaddi, A., Cicero, A., Poli, A., \& Paoletti, R. (2007). Practical guidelines for familial combined hyperlipidemia diagnosis (Vol. 3). Vasc Health Risk Manag.

Goldstein, J., Schrott, H., Hazzard, W., \& Bierman, E. (1973). Hyperlipidemia in coronary artery disease. II. Genetic analysis of lipid levels in 176 families and delineation of a new inherited disorder, combined hyperlipidemia (Vol. 52). J Clin Invest.

Goldstein, J., Schrott, H., Hazzard, W., Bierman, E., \& Motulsky, A. (1973). Hyperlipidemia in coronary heart disease. II: genetic analysis of lipid levels in 176 families and delineation of a new inherited disorder, combined hyperlipidemia (Vol. 52). J Clin Invest.

Grundy, S., Chait, A., \& Brunzell, J. (1987). Familial combined hyperlipidemia workshop (Vol. 7). Arteriosclerosis.

Keulen, E., Kruijshoop, M., Schaper, N., \& et al. (2002). Increased intima mediathickness in familial combined hyperlipidemia associated with apoB (Vol. 22). Arterioscler Thromb Vasc Biol.

Mancia, G., Fagard, R., Narkiewicz, K., Redón, J., Zanchetti, A., Böhm, M., \& et al. (2013). ESH/ESC Guidelines for the management of arterial hypertension:. En ESH/ESC, The Task Force for the management of arterial hypertension of the European Society of Hypertension (ESH) and of the European Society of Cardiology(ESC) (Vol. 31, págs. 1281-357). J Hypertens.

Mata, P., Alonso, R., Ruíz-Garcia, A., Díaz-Díaz, J., \& González, N. (2020). Hiperlipidemia familiar combinada: documento de consenso. Semergen, 40(7), 374-380. doi:http://dx.doi.org/10.1016/j.semerg.2014.07.007

Mc Neely, M., Edwards, K., Marcovina, S., Brunzell, J., Motulsky, A., \& Austin, M. (2001). Lipoprotein and apolipoliprotein abnormalities in familial com- 
bined hyperlipidemia (Vol. 159). Atherosclerosis.

Porkka, K., Nuotio, I., Pajukanta, P., Ehnholm, C., Suurinkeroinen, L., Syvänne, K., \& et al. (1997). Phenotype expression in familial combined hyperlipidemia (Vol. 133). Atherosclerosis.

Skoumas, J., Papadimitriou, L., Pitsavos, C., Masoura, C., Giotas, N., Chrysohoou, C., \& et al. (2007). Metabolic syndrome prevalence and characteristics in Greek adults with familial combined hyperlipidemia (Vol. 56). Metabolism.

Sniderman, A., Castro-Cabezas, M., Ribalta, J., Carmena, R., De Bruin, T., \& De Graaf, J. (2002). proposal to redefine familial combined hyperlipidemia-Third workshop on FCHL (Vol. 32). Eur J Clin Invest.
Valles, V., Aguilar-Salinas, C., Gómez-Pérez, F., Rojas, R., Franco, A., Olaiz, G., . . . Sepúlveda, J. (2002). Apolipoprotein B and Al distribution in the Mexican urban adults: Results of a Nation-Wide Survey (Vol. 51). Metabolism.

Zamora, M., Aguilar-Salinas, C., \& Gomez-Pérez, F. (2004). Prevalencia del síndrome metabólico en la hiperlipidemia familiar combinada (Vol. 12). Rev Endocrinol Nutr.

\section{CITAR ESTE ARTICULO:}

Vicuña Terán, V., Chumbi Zumba, P., León García, J., \& Sánchez Pincay, K. (2020). Manejo de hiperlipidemia familiar combinada. RECIMUNDO, 4(1), 423433. doi:10.26820/recimundo/4.(1).enero.2020.423-433 